\title{
Valve therapy in patients with emphysematous type of chronic obstructive pulmonary disease (COPD): from randomized trials to patient selection in clinical practice
}

\author{
Arschang Valipour \\ Department of Respiratory and Critical Care Medicine, Ludwig-Boltzmann-Institute for COPD and Respiratory Epidemiology, Otto-Wagner- \\ Spital, Sanatoriumsstrasse 2, 1140 Wien, Austria \\ Correspondence to: Arschang Valipour, MD, FCCP, Assoc. Professor. Department of Respiratory and Critical Care Medicine, Ludwig-Boltzmann-Institute \\ for COPD and Respiratory Epidemiology, Otto-Wagner-Spital, Sanatoriumsstrasse 2, 1140 Wien, Austria. Email: arschang.valipour@wienkav.at.
}

\begin{abstract}
In recent years a number of endoscopic methods have emerged to treat patients with severe emphysematous type of chronic obstructive pulmonary disease (COPD), who are primarily symptomatic due to hyperinflation despite optimal medical management. Of these techniques, implantation of endobronchial one-way valves into targeted airways of isolated emphysematous lobes appears to be one of the most promising innovations. Results from randomized controlled trials of valve therapy for emphysema show consistent benefits in terms of lung function, exercise capacity, symptoms, and quality of life. This review aims to provide a comprehensive summary of the currently available scientific data, discussion of typical treatment related side effects, and recommendations for patient selection in clinical practice.
\end{abstract}

Keywords: Emphysema; bronchoscopy; endobronchial valves; lung volume reduction (LVR)

Submitted Jan 20, 2018. Accepted for publication Aug 16, 2018.

doi: $10.21037 /$ jtd.2018.08.86

View this article at: http://dx.doi.org/10.21037/jtd.2018.08.86

\section{Introduction}

Subjects with severe emphysema remain significantly disabled despite optimum medical therapy, pulmonary rehabilitation and long-term oxygen therapy. These therapies, however, have limited ability to reverse or remove the hyperinflation caused by the alveolar destruction. Lung volume reduction surgery (LVRS) involves resection of areas of diseased lung to reduce hyperinflation and to improve breathing mechanics (1). The National Emphysema Treatment Trial (NETT) study demonstrated that this procedure can provide clinical benefits in selected subject groups: subjects with predominantly upper lobe disease and low-exercise capacity (2). However, surgical Lung volume reduction (LVR) is associated with significant morbidity and mortality with a 3 -month postoperative mortality rate of $5-10 \%$ and a nonfatal complication rate of $60 \%$ (3). Other articles in this issue of the journal will focus in more depth on appropriate patient selection and outcomes of LVRS.

Since the publication of the NETT study results a number of different, potentially less invasive methods have been studied, to achieve the clinical benefits of surgical LVR with a better safety profile. Of these, bronchoscopic LVR through the implantation of one-way valves has the largest body of scientific data available to date. The present review will critically discuss the currently available literature related to valve therapy, some procedural aspects, and the potential impact of both for clinical practice.

\section{Valve technology}

Two valve systems have been designed to ensure volume reduction and at the same time accommodate drainage of mucus. The first is the Zephyr Endobronchial Valve (EBV) system (Pulmonx Inc., Palo Alto, Calif., USA). It is constructed from biocompatible materials and is easy to deploy and remove. It has an outer cylindrical frame with a circumferential wire mesh and a central lumen that anchors a duck bill-shaped one-way valve. Different sizes 

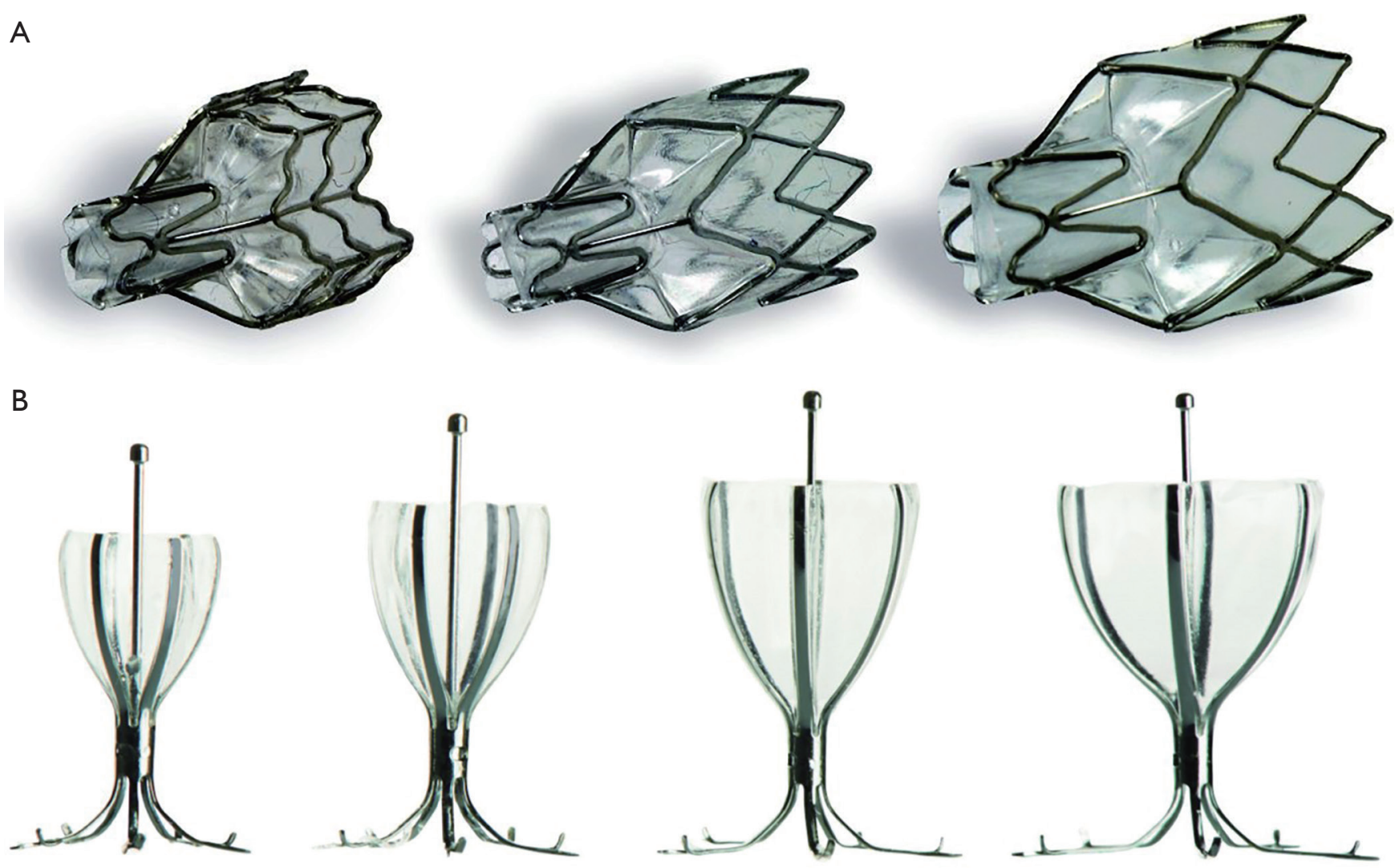

Figure 1 Endobronchial valves (Zephyr valve system, Pulmonx) (A) and intrabronchial valves (Spiration valve system, Spiration/Olympus) (B).

and lengths of the valves are available to ensure lobar occlusion, despite variable airway anatomy leading to the target lobe (Figure 1A). Spiration/Olympus (Spiration Inc., Redmond, Wash., USA) manufactures the second valve system, the Intrabronchial Valve (IBV). This system has an umbrella design in which an elastomer covering is stretched over a nitinol wire frame that anchors the device in place (Figure $1 B$ ). During breathing, expiratory flow under pressure can escape from the lung around the edges of the flexible covering, but is prevented from flowing in the forward direction. Similar to the EBV, the IBV is available in multiple sizes and deployed into the airways through a flexible bronchoscope using a delivery catheter, and is easy to remove.

\section{Pathophysiologic impact of valve therapy}

The aim of valve treatment is to block the inspiratory airflow into targeted, hyperinflated regions of the lung, while allowing air to escape upon exhalation (4). Reducing the overall size of the hyperexpanded emphysematous lung produces space within the less compliant chest cavity for the remaining lung to expand and thereby improving overall lung mechanics and respiratory muscle function during inspiration. Figure 2 shows X-rays from a patient who underwent valve treatment in the left upper lobe, with subsequent development of left upper lobe atelectasis. Reducing hyperinflation through valve therapy has been shown to be associated with improvements in chest-wall asynchrony (5), and a reduction in the work of breathing translating into increased exercise capacity (6). The latter observation is most likely due to airflow redistribution into areas of the lung with better elastomechanical properties, thereby reducing not only static, but also dynamic hyperinflation during exercise $(6,7)$. Endobronchial blockade with one-way valves is furthermore associated with a reduction in regional perfusion. Pizarro et al. (8) studied 26 patients with heterogeneous emphysema who underwent EBV therapy due to severe symptomatic hyperinflation. Lung scintigraphy was done prior to valve therapy and eight weeks thereafter. Target zone perfusion showed a mean relative reduction of $43 \%$ and was associated with 


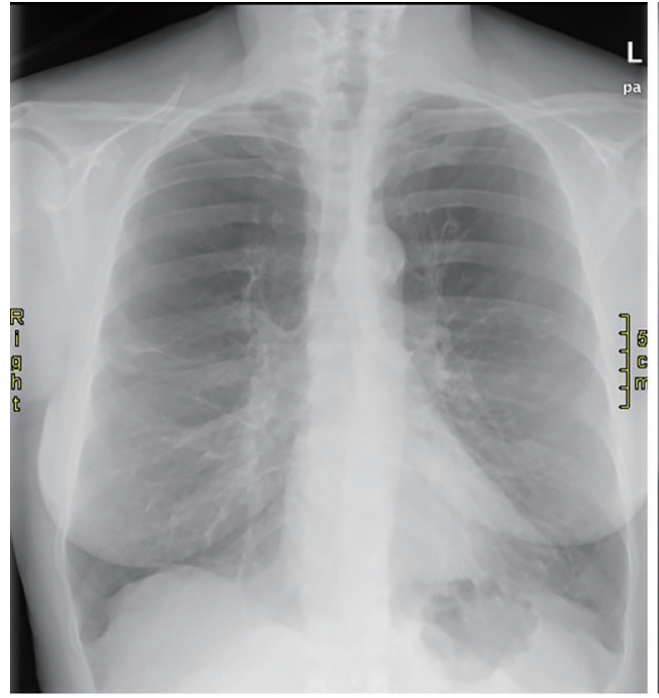

Pre valve treatment

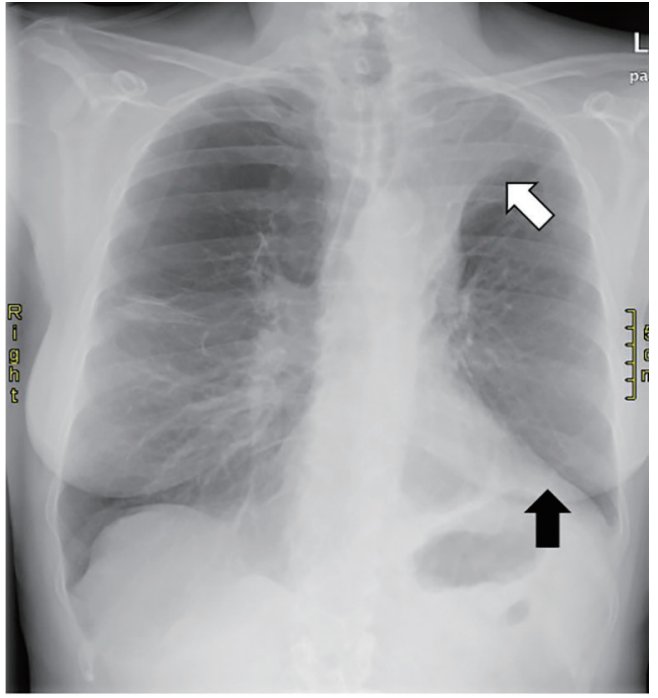

Post valve treatment

Figure 2 Pre- and post-procedural X-rays from a patient who underwent valve therapy for severe emphysema. The left image shows the preprocedural X-ray from a female patient with severe emphysema. The right image shows the X-ray obtained 1 week after valve therapy in the left upper lobe. Note evidence of left upper lobe atelectasis (white arrow) and an elevated diaphragm (black arrow). The patient responded favourably to the treatment with an improvement in her symptoms, lung function, and exercise capacity.

perfusional redistribution to untreated areas of the lung.

Benefits of volume reduction, however, extend beyond improvements in respiratory function. In a pilot study with a sample size of six patients, Eberhardt and colleagues (9) reported a decrease in mean pulmonary artery pressure and pulmonary capillary wedge pressure using right heart catheterization prior to and at 90 days post successful valve therapy. The hemodynamic changes were further associated with improvements in cardiac index, an observation consistent with effects of surgical LVR on cardiovascular function (10). A similar observation was made by Pizarro et al., who demonstrated improved right ventricular function using echocardiography before and after 8 weeks following valve treatment in patients with severe emphysematous type of chronic obstructive pulmonary disease (COPD) (11).

Improvements in cardiovascular function after effective LVR may be due to improvements in preload and reductions in the afterload, therefore promoting improved cardiac function (10). Furthermore, there is evidence of a more effective work of breathing after LVR, which may lead to an attenuation of oxygen consumption of respiratory muscles (12). It has also been hypothesized that the increase in cardiac index is in part due to capillary recruitment of the previously compressed lung zones (13).

\section{Early phase clinical trials and predictors of success}

The first randomized controlled trial (RCT), the Endobronchial Valve for Emphysema Palliation Trial (VENT) prospectively studied the efficacy and safety of EBV therapy (14). The study had a US cohort and a European cohort. Eligibility criteria included a diagnosis of heterogeneous emphysema (based on quantitative and visual indices of lobar emphysema severity using the NETT scoring criteria), a forced expiratory volume in $1 \mathrm{~s}\left(\mathrm{FEV}_{1}\right)$ of $15-45 \%$ predicted, and evidence of hyperinflation with a total lung capacity of more than $100 \%$ and a residual volume (RV) of more than $150 \%$ predicted despite optimized medical management. Before randomization, patients underwent 6-8 weeks of pulmonary rehabilitation. High-resolution computed tomography (HRCT) performed at baseline and at 6 months was used to determine eligibility as well as treatment outcomes. All images were analyzed at a core laboratory. The target lobe was the one with the most emphysematous destruction and the most severe hyperinflation. In the US cohort of this study, 220 patients were randomized to valve therapy and were compared with 101 patients randomised to a control group that 
continued to receive standard medical care (14). Primary end points were changes in $\mathrm{FEV}_{1}$ and 6-minute walking distance (6MWD). A total of 820 valves were inserted in the treatment group (median 4, range 1-9 per patient). Results showed statistically significant improvements in lung function, exercise tolerance and quality of life, however, failed to meet criteria for the minimal clinically important difference (MCID) for these outcomes. The FEV improvement of the treatment arm was $4.3 \%$ as compared with a decrease of $2.5 \%$ in the control group, resulting in a mean between-group difference of $6.8 \%$.

Two main factors have contributed to the lack of clinical benefits in response to valve therapy in this study:

(I) Collateral ventilation: In the context of valve therapy, it is characterised as ventilation between adjacent lobes of the lung, a phenomenon both present in normal human lungs and in patients with emphysema (15). The reported prevalence of incomplete fissures is as high as $85 \%$ for the right major fissure, $74 \%$ for the left major fissure, and $90 \%$ for the minor fissure (16). As a result, when endobronchial valves are placed, lobar volume reduction is not achieved as air is still able to enter the lobe via interlobar collateral pathways. Incomplete interlobar fissures detected on a highresolution scan may be indicative for the underlying parenchymal fusion between adjacent lobes, and consequently predict collateral ventilation via channels that bypass the usual airways. However, as the impact of collateral ventilation on the outcome of valve therapy was not appreciated at the time of designing the VENT study, both patients with and without collateral ventilation were included;

(II) Lobar occlusion: of those patients who underwent a 6 months follow-up CT scan in the VENT study, $44 \%$ were found to have incomplete lobar occlusion due to malpositioning, expectoration, and/or migration of at least one valve (14). Incomplete lobar occlusion with valves allows air to enter upon inspiration, thus preventing lobar volume reduction in the presence of intersegmental collateral ventilation.

When these two major criteria (complete fissures and lobar occlusion) were met, the minimally clinically important difference thresholds for $\mathrm{FEV}_{1}$, cycle ergometry workload, 6MWD and St. George's Respiratory Questionnaire (SGRQ) based on an analysis of the European cohort of the VENT study were met by $67 \%$,
$44 \%, 56 \%, 67 \%$ of emphysema patients at 12 months, respectively (17). Importantly and in contrast to the NETT study results, valve therapy appears to be equally effective in upper and lower lobe predominant emphysema (18).

The above-mentioned predictors for treatment success are similarly responsible for the absence of clinically meaningful outcomes in the randomized multicenter, shamcontrolled European IBV treatment study (19). Patients with upper lobe predominant severe emphysema were randomised to bronchoscopy with $(n=37)$ or without $(n=36)$ IBV valves for a 3-month blinded phase. Per protocol, one segment or sub-segment of the right upper lobe and the lingula segments of the left upper lobe were not treated to achieve incomplete occlusion of the upper lobes and to prevent lobar atelectasis. The aim of this treatment strategy was to shift the ventilation to adjacent untreated lobes, but avoiding full lobar atelectasis, due to an increased risk of complications observed with atelectasis in an earlier pilot study (20). In the European IBV study a mean $7.3 \% \pm 9 \%$ lobar volume reduction evaluated by CT volumetry was achieved, which did not translate into clinically meaningful improvements in lung function, symptom scores, exercise capacity, or arterial blood gas analysis.

In a direct comparison of the above mentioned two strategies, Eberhardt and colleagues (21) previously confirmed superiority of unilateral lobar occlusion over bilateral segmental upper-lobe treatment with incomplete lobar occlusion. In this prospective, randomized, singlecenter study, 22 patients were randomized, 11 patients treated with IBV in each arm. Both at 30 and 90 days, significant differences were seen in lung function, symptom scores, and exercise capacity, in favor of unilateral treatment.

\section{Assessing collateral ventilation}

The assessment of collateral ventilation can currently by achieved via two different methods, CT-based assessments of fissure integrity and real-time flow measurement-based recordings of collateral ventilation using the Chartis system.

Thoracic HRCT scan, which has been used for detection of emphysema severity and disease distribution, can further be used to assess the integrity of interlobar fissures. Visual evaluation of fissure completeness requires assessment of fissure continuity on multiple planes of chest CT scan reconstructions, a time-consuming process with considerable inter-reader variability, particularly with respect to the minor fissure (22). In fact, it has previously 


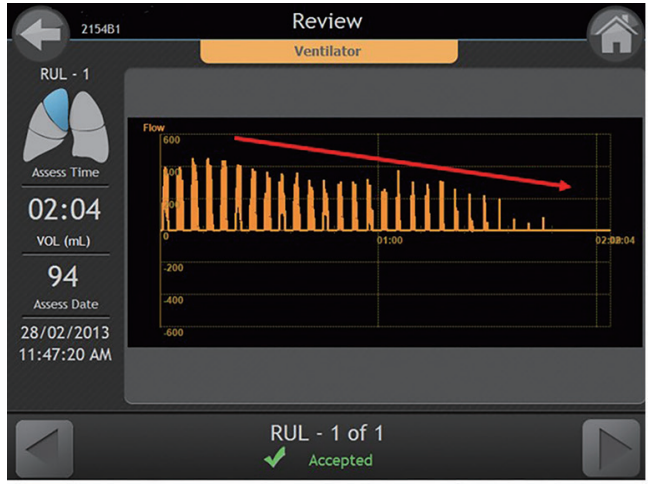

Collateral ventilation negative

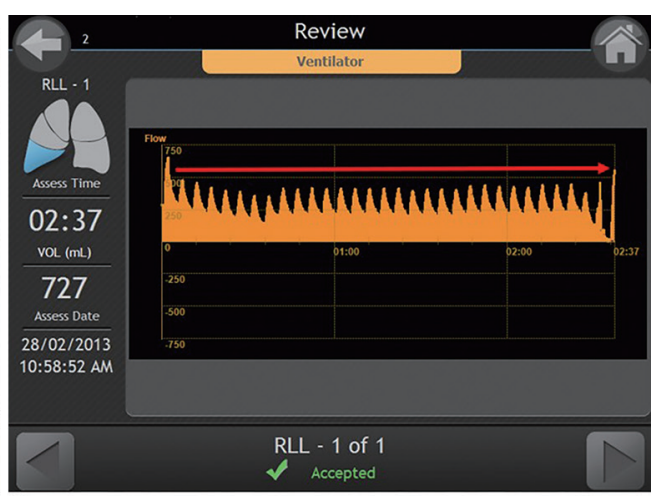

Collateral ventilation positive

Figure 3 Chartis measurements during mechanical ventilation. The left image demonstrates a Chartis recording of a patient without collateral ventilation, characterized by a gradual reduction in target lobar flow. The right image demonstrates a Chartis recording of a patient with persistent flow in the target lobe, despite occlusion with a balloon, indicating collateral ventilation.

been demonstrated that there is only little agreement between chest physicians and experienced radiologists in visual fissure analysis (22). Alternatively, automated computer-based schemes assessing fissure integrity have been described and tested $(23,24)$. These measurements are potentially more robust, precise, and reproducible. On the other hand, they usually require specific software, with significant measurement variations for emphysema between different software tools (25).

Real-time assessment of collateral ventilation can be achieved via an endoscopic catheter-based technique (26). The Chartis Pulmonary Assessment System consists of a single-patient-use catheter with a compliant balloon component at the distal tip, which upon inflation blocks the airway. Air can then flow out from the target compartment into the environment through the Chartis catheter's central lumen, and is displayed on a connected console. Airway resistance can be calculated and collateral ventilation identified in isolated lung compartments. The assessment can be performed during spontaneous breathing or mechanical ventilation under general anesthesia. With increasing experience, measurement artifacts are observed in as little as $8 \%$ of recordings, thus providing physicians a reliable tool for the detection of collateral ventilation (27). A flow reduction below $20 \%$ of baseline measurements is being considered the threshold for the exclusion of clinically meaningful collateral ventilation (27) (Figure 3). Clinical applicability of the Chartis System was prospectively investigated in a prospective multicenter trial of 96 patients (of which only 80 were available for final analysis) (28). In this study, patients without collateral ventilation demonstrated a median target lobar volume reduction (TLVR) of $752 \mathrm{~mL}$ and a mean $16 \% \pm 22 \%$ $\mathrm{FEV}_{1}$ improvement from baseline, together with quality of life improvements (SGRQ: -10 points) at 30 days postprocedurally. Expectedly, there was no significant TLVR and no clinical benefits in patients with collateral ventilation. Overall, the Chartis assessment was able to predict the response to valve therapy with $71 \%$ accuracy.

In a direct comparison of both techniques, HRCT fissure analysis and the Chartis System were found to be equally effective for predicting the response to valve therapy (29). According to a study by Gompelmann et al. (30) the Chartis method had an accuracy of $74 \%$ and fissure analysis an accuracy of $77 \%$, when using a TLVR of $350 \mathrm{~mL}$ or greater as a surrogate of treatment success. Both methods were concordant in two-thirds of the patients, thus a certain proportion of patients may require assessment with both techniques to provide a high likelihood of treatment response.

\section{RCTs in patients with low collateral ventilation}

The following RCTs conducted with the EBV subsequently examined the efficacy of valve therapy only in those with low or absent collateral ventilation. In the "Bronchoscopic lung volume reduction with endobronchial valves for patients with heterogeneous emphysema and intact interlobar fissures" (BeLieVeRHiFi) study, patients with complete fissures on CT $(n=25)$ were treated with valve therapy and were compared to a control group that received sham bronchoscopy without valve placement $(n=24)(31)$. 
The primary endpoint was the percentage change in postbronchodilator $\mathrm{FEV}_{1}$ measured 90 days postprocedure. Four treated patients, despite having complete fissures on $\mathrm{CT}$ as a criterion for study entry, had collateral ventilation detected by the Chartis system. The data for these patients were included in the intention-to-treat analysis. In the treatment group $\mathrm{FEV}_{1}$ increased by a mean of $24.8 \%$ (median $8.7 \%$ ) in the valve group and $3.9 \%$ in the control group at 3 months. This result was accompanied by significant improvements in lung volumes and exercise capacity, however, symptom improvements using COPD Assessment Test (CAT) and SGRQ were not statistically significant between the groups. Importantly, improvements in exercise capacity were associated with treatment related improvements in spirometry $\left(\mathrm{FEV}_{1}\right)$ and lung volumes. A follow-up report including patients from the control group crossing over to valve treatment confirmed the results reported in the initial treatment arm, with a mean $27 \%$ $\mathrm{FEV}_{1}$ improvement (32).

In the STELVIO (Endobronchial Valves for Emphysema without Interlobar Collateral Ventilation) trial, eightyfour patients were screened and underwent baseline bronchoscopy (33). Of these patients, 16 were excluded because they had collateral ventilation or because the airway anatomy was not suitable for valve placement, resulting in a total of 68 patients who were randomly assigned to a valve treatment group $(n=34)$ or control group $(n=34)$. A median of 4 endobronchial valves (range, 2-7) were placed per patient, with a median procedure time of 18 minutes (range, 6-51 minutes). In the intention-to-treat population, improvements in $\mathrm{FEV}_{1}$ (mean $20.9 \% \mathrm{EBV} v s$. $3.1 \%$ control), forced vital capacity, and 6-minute walk test (6MWT) were significantly greater in the valve treatment group compared with the control group. Noteworthy, repeated bronchoscopy was deemed necessary in $35 \%$ of patients in the EBV group, due to permanent valve removal because of recurrent pneumothorax, changes in airway anatomy after atelectasis, pneumonia or lack of efficacy. Outcomes were expectedly of larger magnitude in the 25 EBV treated patients who completed the study, with mean improvements in exercise capacity of 106 meters in the 6MWT and -14.7 points in the SGRQ. Patients in the control group who crossed over to valve treatment at 6 and 12 months had similarly improved COPD outcomes (34).

The TRANSFORM study was a prospective, multicenter 2:1 RCT of EBVs plus standard of care versus standard of care alone (35). Primary outcome was the percent of patients with a $\mathrm{FEV}_{1}$ improvement from baseline of $12 \%$ or greater at 3 months post-procedure. Ninety-seven subjects were randomized to EBV $(n=65)$ or standard of care $(n=32)$. At 3 months, $55.4 \%$ of EBV and $6.5 \%$ of SoC subjects had an $\mathrm{FEV}_{1}$ improvement of $12 \%$ or greater. Improvements were maintained with patients in the EBV group demonstrating a $20.7 \% \pm 29.6 \%$ increase in $\mathrm{FEV}_{1}$ at 6 months compared with baseline. The mean TLVR derived from HRCT analysis was $1.09 \pm 0.62 \mathrm{~L}$, which translated in significant reductions in $\mathrm{RV}$ measured using body plethysmography $(-700 \mathrm{~mL}$ $\triangle \mathrm{EBV}-\mathrm{SoC})$.

The recently published LIBERATE trial is the first multicentre RCT in patients with heterogeneous emphysema that provides follow-up data at 12 months post-EBV therapy (36). The primary outcome was the responder rate of treated patients $v s$. controls with a $\geq 15 \%$ post-bronchodilator $\mathrm{FEV}_{1}$ improvement from baseline. In fact, at 12 months $48 \%$ of EBV treated patients fulfilled this criterion, the responder rate in controls was $17 \%$. Consistent with above mentioned studies, there were statistically and clinically meaningful improvements in exercise capacity, quality of life, and hyperinflation. In a previously unreported analysis supplemental oxygen usage at 12 months was evaluated postprocedurally. A larger proportion of EBV subjects compared to SoC (16\% vs. 7\%, respectively) used less oxygen whereas a larger proportion of SoC subjects compared to EBV (22\% vs. $11 \%$, respectively) used more oxygen at 12 months as compared to their baseline usage. In the majority of patients, however, there was no substantial change in oxygen usage, despite substantial improvements in hyperinflation related symptoms.

The IMPACT study was the first major prospective trial to evaluate the efficacy and safety of EBV in patients with exclusively homogeneous emphysema and absence of collateral ventilation (37). For patients with severe homogeneous emphysema, treatment options are limited in general. LVRS is not recommended in this specific cohort of patients due to its variable success and increased mortality risk (2). As subgroup analysis from previous reports $(17,33)$ indicated beneficial outcomes for valve therapy independent of interlobar heterogeneity, the IMPACT study was designed as a prospective, multicenter 1:1 RCT of valve therapy versus standard of care. The primary outcome was the percentage change in $\mathrm{FEV}_{1}$ at 3 months relative to baseline in the EBV group vs. the standard of care group. Secondary outcomes included changes in $\mathrm{FEV}_{1}$, SGRQ, 6MWD and target lobe volume reduction. Collateral ventilation was assessed using the Chartis System. Potential 
subjects underwent HRCT to confirm homogeneity in a core-lab using computerized software. Only those subjects with a heterogeneity index (difference in destruction scores between potential target and ipsilateral lobes) below $15 \%$ were considered eligible for the study. A potential target lobe was identified on the basis of the highest destruction score and lowest perfusion using perfusion scintigraphy. Qualifying subjects were randomized at a 1:1 ratio into the EBV treatment or no EBV treatment arm. Both groups continued to receive standard of care treatment for COPD. The results demonstrated statistically significant and clinically meaningful improvements in lung function, exercise capacity and quality of life in EBV-treated patients versus the standard of care only group. There was a mean \pm standard deviation between group difference for the change in $\mathrm{FEV}_{1}$ of $18.8 \% \pm 22.1 \%$, 40-meter difference in $6 \mathrm{MWD}$, and a 10-point difference in SGRQ, both in favor of the EBV group.

Table 1 provides responder rates for the above mentioned RCTs in the per-protocol population, i.e. for patients who were treated with valves and were available for follow-up assessments.

In addition to the above-mentioned studies with the EBV, a RCT utilizing the IBV System in patients with severe emphysema and complete fissures has been presented recently (38). The REACH study included 101 patients who were randomized 2:1 to receive either IBV valves (with the intention of achieving lobar occlusion) or optimal medical management. Statistically significant and clinically meaningful improvement in mean $\mathrm{FEV}_{1}$ were observed for the treatment group at the 1-, 3-, 6- and 12-month visits (16.8\%, 14.2\%, 20.7\%, 22.8\%). Significant improvements were also observed for quality of life measures and $6 \mathrm{MWD}$, however, further information is lacking at this moment due to pending publication of the manuscript.

\section{Safety of valve therapy}

The most common severe complications of endobronchial valve placement include pneumothorax, COPD exacerbations, pneumonia, and/or valve dislocations.

\section{Valve associated pneumothorax}

The pneumothorax rate associated with valve treatment is between $20 \%$ and $30 \%$ according to the most recent clinical trials (33,35-37). As every pneumothorax can be life threatening in these patients with limited respiratory reserve, there is a need for a standardized pneumothorax management in this particular patient population. An algorithm for the management of valve associated pneumothorax has been previously published, which provides a useful guidance for physicians performing valve therapy (39). These recommendations outline a strategy depending on the size and clinical impact of the pneumothorax, involving valve removal in the presence of prolonged air leak and subsequent medical and/or surgical pneumothorax management. As the large majority of pneumothorax events occur within the first 48 hours and require chest tube placement, current practice in many institutions is to admit patients to hospital for at least 5 days following insertion of valves for emphysema. Particularly, patients with significant volume reduction on post procedure chest $\mathrm{X}$-rays and those with preprocedural pleural adhesions (in the adjacent untreated lobe) may present a higher risk of pneumothorax (40). Patients should be instructed to report to medical institutions immediately in the presence of signs and symptoms of a pneumothorax. Herzog and co-workers (41) recommend limiting physical activity with bed rest and pharmaceutical cough suppression within the first 48 hours of valve therapy to reduce the periinterventional pneumothorax event rate. The authors hypothesized that biomechanical forces during breathing (during exertion) and coughing may promote the development of pneumothorax. In a retrospective study they were able to observe a lower pneumothorax risk $(5 \%)$ with the above mentioned modified postinterventional approach, when compared with standard medical care $(25 \%)$, that did not include specific restrictions on physical activity or cough suppression. Given the magnitude of difference in pneumothorax risk observed and the potential clinical relevance, these findings need to be formally replicated in a prospective, randomized study, before introducing them into routine clinical care.

\section{Lower respiratory tract infections and COPD exacerbations}

Prophylactic antibiotics and a short course of systemic steroids has been obligatory throughout all clinical trials of valve therapy, and is being continued as part of standard of care at most institutions since then. Despite this pharmaceutical strategy, up to $20 \%$ of patients develop lower respiratory tract infections within the first three months after the procedure, manifesting as acute COPD exacerbations, bronchitis, and/or pneumonia events 
Table 1 Responder rates in the valve treatment group based on Chartis assessment in patients with severe, emphysematous type of COPD (per protocol population for all studies, intention-to-treat analysis for the Liberate study)

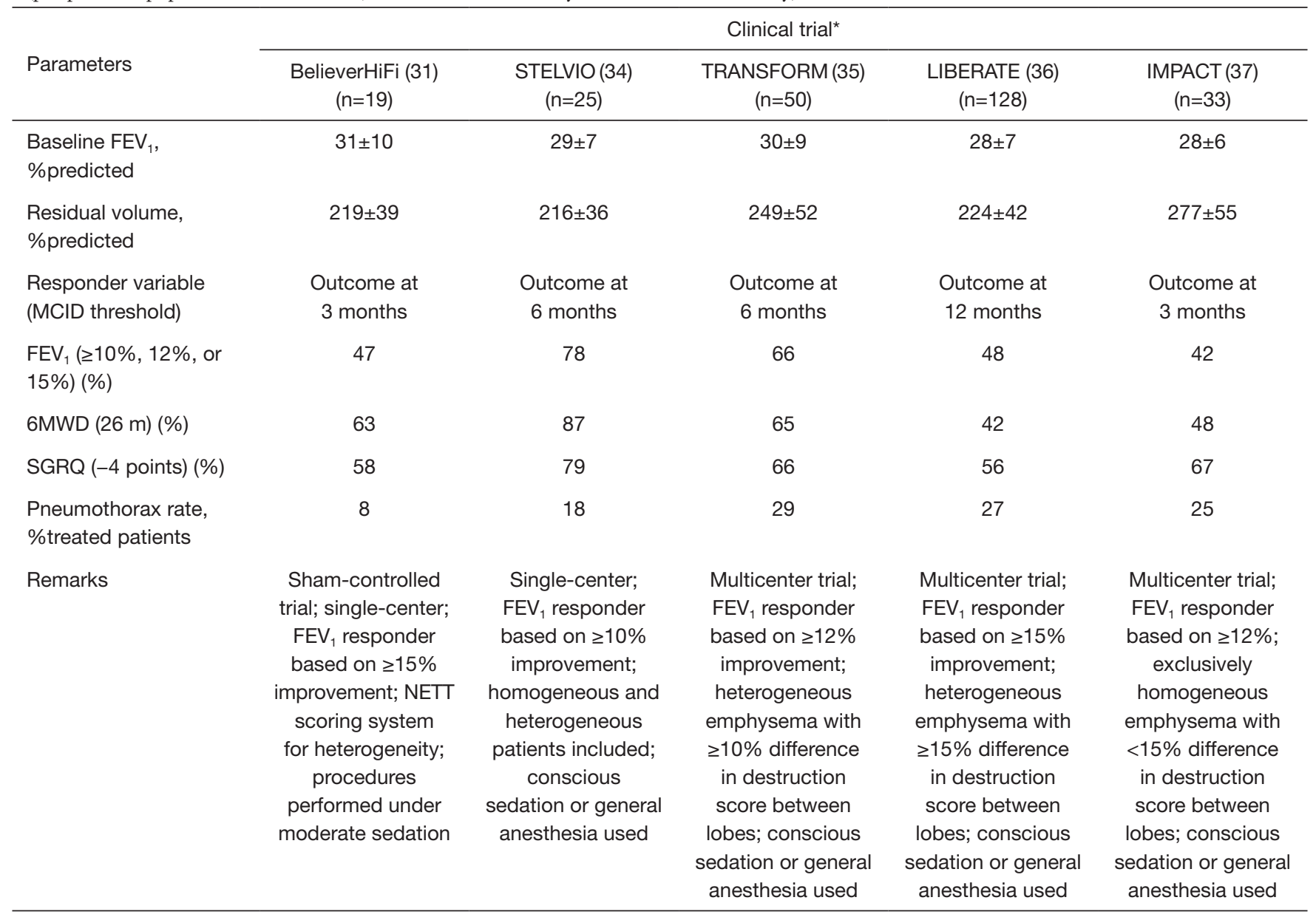

In all of the above-mentioned studies statistically significant differences were observed compared with responder rates in controls. *, patient numbers $(\mathrm{N})$ for outcomes based on available results for $\mathrm{FEV}_{1}$ responder data. MCID, minimal clinical important difference; $\mathrm{FEV}_{1}$, forced expiratory volume in 1 s; 6MWD, 6-minute walking distance; SGRQ, St George's Respiratory Questionnaire.

$(31,33,35,37)$. While the true origin of these events is not entirely clear, recent studies have provided evidence of bacterial growth in the presence of endobronchial valves, which were detected during sampling of secretions at follow-up bronchoscopies (42). It remains yet to be established whether these microorganisms are responsible for the above-mentioned adverse events or whether they should be considered colonisation without clinical impact. However, in the presence of repeated exacerbations and/ or postobstructive pneumonia, removal of valves should be considered until full clearance of the infection and clinical stability is ensured. Replacing valves may be an option after careful risk-benefit assessment and patient shared decision making.

\section{Valve dislocation and migration}

Valve dislocation is rare and usually associated with inappropriate sizing and/or placement. It should be suspected in patients with sudden loss of efficacy and/or in the presence of a severe intractable cough. A chest CT scan with or without bronchoscopy may be necessary to confirm the correct position of the valves in the target region. Valve replacement may be necessary in more distal airways to ensure sufficient (lobar) occlusion.

\section{Patient selection in clinical practice today}

In order to be considered eligible for valve therapy, a number of selection criteria should be applied prior to 
Table 2 Patient selection criteria for valve therapy in emphysema (based on Slebos et al.) (43)

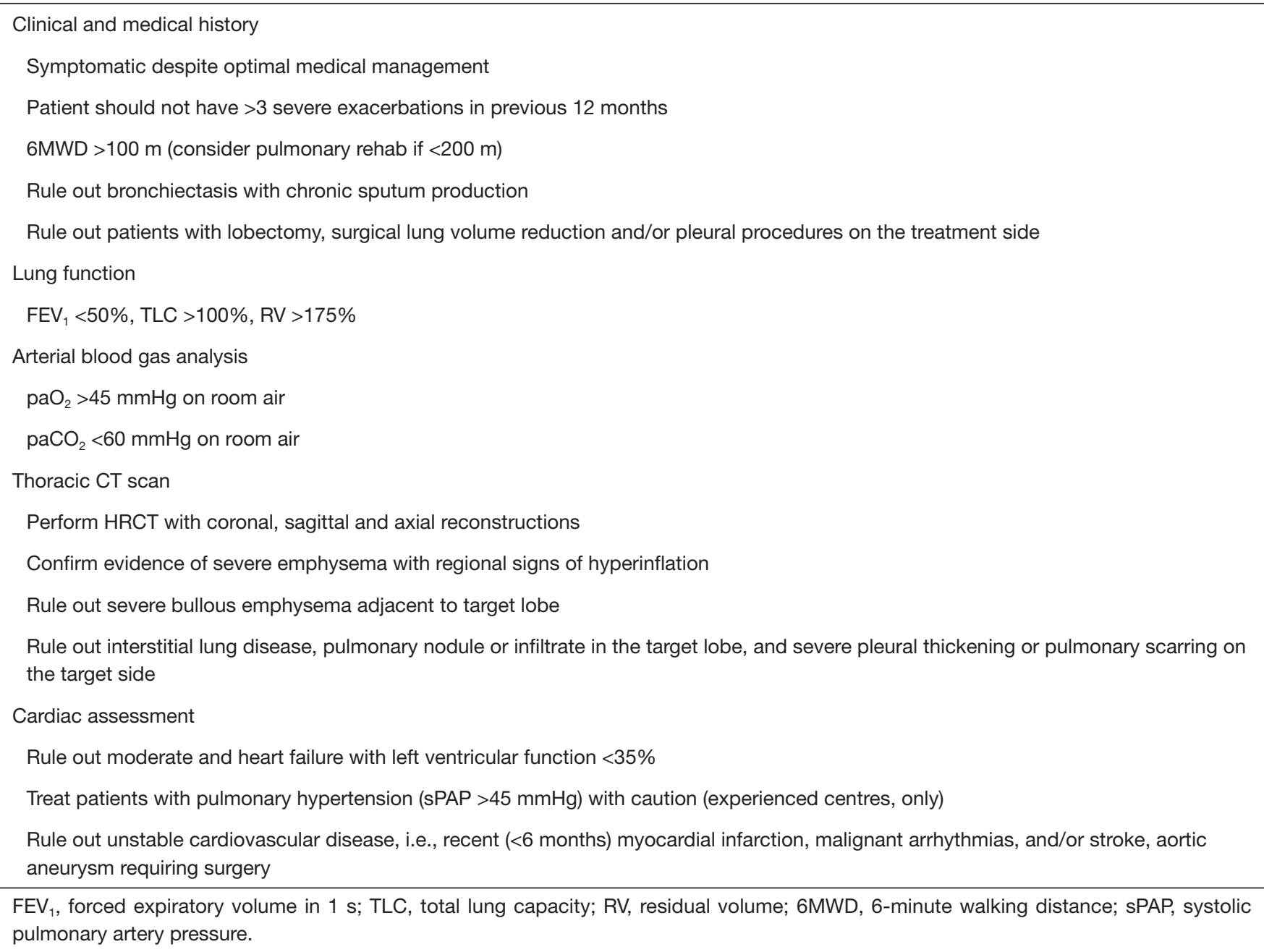

potential valve placement (Table 2). For more information please refer to a recent standard of practice review, which provides a more in-depth review of the below eligibility criteria and procedural aspects (43).

\section{Medical bistory and comorbidities}

A prerequisite for further evaluation of a patient for endoscopic valve therapy is optimum medical management of the underlying disease per national or international guidelines. This includes, but is not limited to maximum inhaler therapy, ensuring treatment adherence, smoking cessation prior to valve therapy, vaccinations, and/or oxygen therapy if required. Patients that are considered clinically unstable despite optimal medical management, with more than three severe COPD exacerbations in the previous 12 months should be excluded until stabilized. Similarly, endoscopic valve therapy should not be performed in patients with symptomatic bronchiectasis and chronic sputum production with microbiological colonization, such as pseudomonas or methicillin-resistant Staphylococcus aureus (MRSA). Patients with unstable cardiovascular disease such as severe heart failure [left ventricular ejection fraction (LVEF) $<35 \%$ despite optimal medical management], unstable cardiac arrhythmia, myocardial infarction or stroke within the past 6 months should also be excluded until stabilized or improved. Although not an absolute contraindication, patients with evidence of pulmonary hypertension and systolic pulmonary artery pressure greater than $45 \mathrm{mmHg}$ (using echocardiography and/or right heart catheter measurement) should be treated with caution at experienced centers. 
Hyperinflation without emphysema

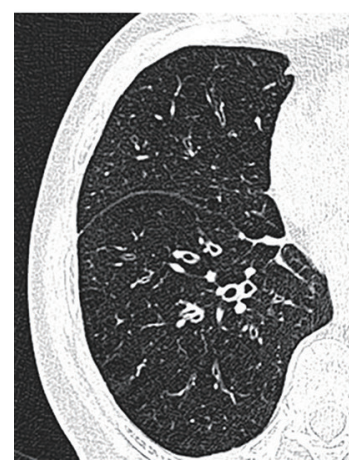

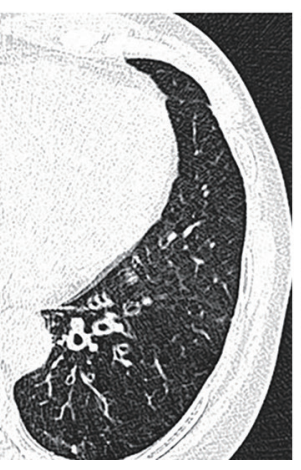

Hyperinflation with emphysema

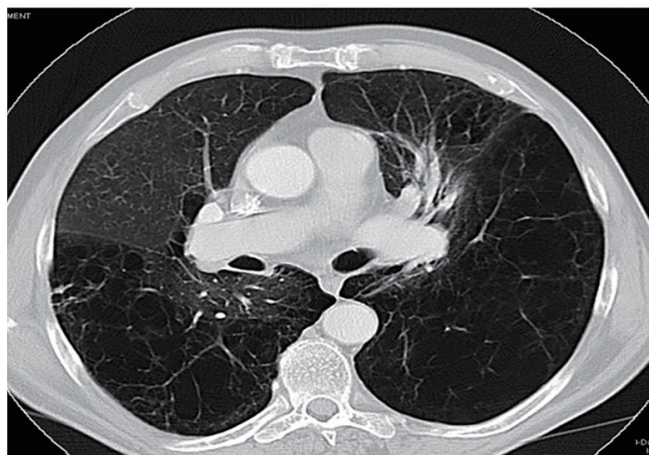

Figure 4 Radiological phenotypes of severe COPD. CT scans from two patients with similar age, gender, body-mass-index, smoking history, lung function impairment, and degree of hyperinflation. However, one CT scan demonstrates severe emphysema (right), while the other scan (left) demonstrates substantial airway wall thickening and some degree of bronchiectasis. COPD, chronic obstructive pulmonary disease.

\section{Surgical bistory}

Patients who have had thoracic surgical procedures, such as bullectomies, lobar, segmental- or wedge resections on the contralateral lung may be potential candidates for valve therapy if they meet the other eligibility criteria. Patients with prior surgery on the same side as the target lobe (including bilateral lung transplant or previous bilateral LVRS), or patients with previous pleurodesis should be excluded due to pleural adhesions and compliance restrictions in the remaining lobes.

\section{Lung function and exercise capacity}

There are no absolute spirometry cut-offs when considering patients for valve therapy. In clinical practice, however, most patients have a post-bronchodilator $\mathrm{FEV}_{1}$ below $50 \%$ and as low as $15 \%$ (44). Since valve therapy works primarily by reducing lung hyperinflation, it is crucial to select patients with evidence of hyperinflation, as measured by a total lung capacity $>100 \%$ and $\mathrm{RV}>175 \%$. While the role of diffusing capacity of the lung for carbon monoxide (DLCO) has been questioned in more recent trials of valve therapy for emphysema, it should be remembered that a diffusion capacity below $20 \%$ of predicted value has been associated with an increased mortality in surgical LVR procedures (2). The periinterventional risk may also be increased in patients with very low baseline exercise capacity, thus expert recommend a trial of pulmonary rehab in patients with a $6 M W T<200$ meters and then reassessment (42).

\section{Arterial blood gas analysis}

Patients with severe hypercapnia ( $>60 \mathrm{mmHg}$ on room air) and/or severe hypoxemia ( $<45 \mathrm{mmHg}$ on room air) should be excluded from valve treatment. Patients with hypercapnia may be reevaluated after at least 3 months of regular noninvasive ventilation and subsequent clinical stability and improvements in $\mathrm{paCO}_{2}$ levels.

\section{Chest CT}

A thin slice (0.5 to $1.5 \mathrm{~mm})$ volumetric HRCT should be performed to evaluate the degree and distribution of emphysema. Figure 4 provides example CT scan images from a patient with and without severe emphysema, despite similar clinical and functional characteristics. Coronal, sagittal and axial reconstructions of the HRCT are useful to determine potential target lobe(s), based on regional signs of hyperinflation, parenchymal destruction, heterogeneity between lobes, and interlobar fissure integrity. When assessing these scans, it should be noted that some patients may exhibit a degree of intralobar segmental heterogeneity (45). In the presence of "preserved" lung tissue segments within a diseased lobe, lobar atelectasis may potentially worsen ventilation perfusion ratio, thus compromising oxygenation levels. Furthermore, the HRCT should be carefully reviewed for findings, such as bulla and/or pleural adhesions in adjacent lobes, but also other pulmonary pathologies, such as suspicious nodules, infiltrates, cavities, severe bronchiectasis, paraseptal emphysema, interstitial lung disease, fibrosis or 


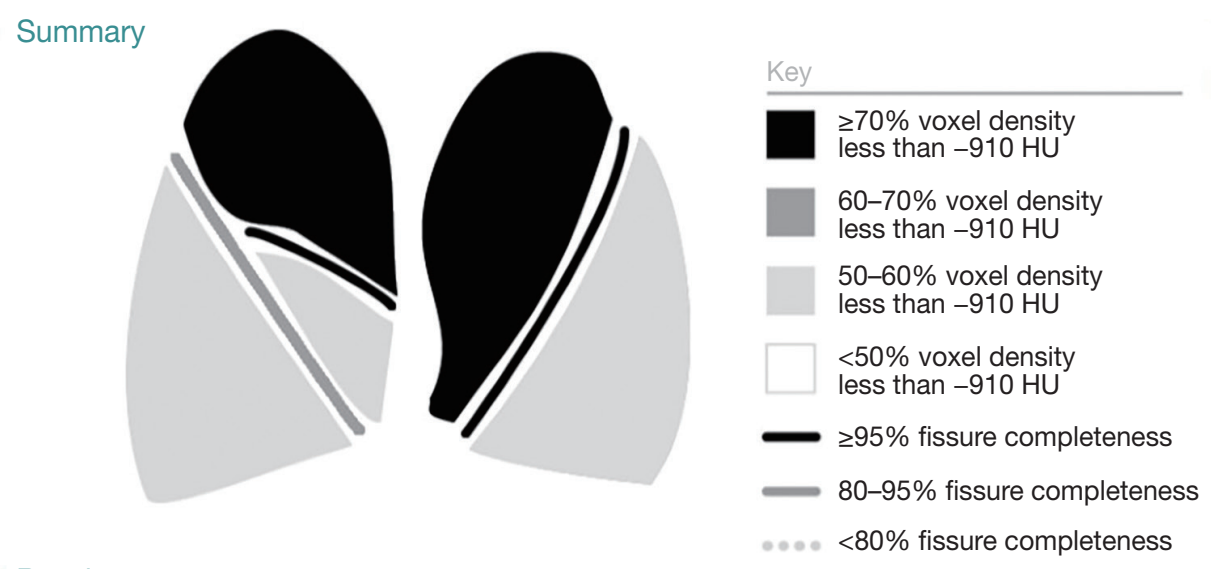

Results

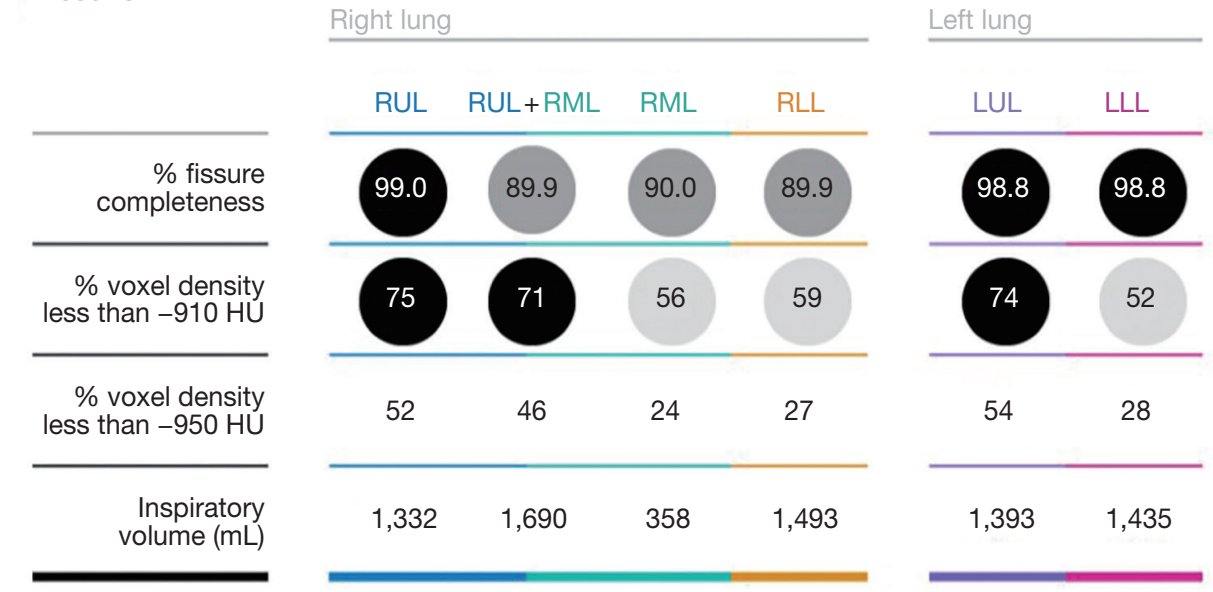

Figure 5 Emphysema quantification and interlobar fissure integrity report. The report indicates severe, bilateral, upper-lobe predominant emphysema with complete major fissures. Based on the report the patient may qualify for valve treatment in either left upper or right upper lobe.

other abnormalities that may be associated with an increased periinterventional risk or poor outcomes (43).

After radiological exclusion criteria have been ruled out by visual assessment, emphysema quantification may be applied to quantify severity and distribution of emphysema beyond the limitations of the naked eye. Multiple software solutions exist to date and both valve producing companies provide a service for analysis of anonymized HRCT scans, to guide physicians in the treatment planning. These reports provide data on lobar volumes, emphysema destruction scores, fissure integrity and heterogeneity indices, based on -910, -920, and/or -950 HU. Figure 5 provides an example report for emphysema quantification (StratX report, Pulmonx). However, it is important to acknowledge that there is substantial inter-software variability with disagreement in the assessments of lobar volumes and emphysema indices between different software protocols applied (46). Thus, it is recommended to keep the scanning protocol and quantification software for longitudinal emphysema monitoring constant.

\section{Perfusion scintigraphy}

The information obtained by perfusion scintigraphy is limited by the methodologically inevitable stratification into craniocaudal lung zones rather than lobes. In patients with multiple potential target lobes, however, perfusion scintigraphy, especially in homogeneous or mild heterogeneous emphysema, may be additionally helpful to identify the target lobe (37). On the other hand, a high perfusion in the untreated ipsilateral lobe is a strong predictor of exercise capacity after valve therapy (47). 


\section{The bronchoscopic procedure}

A treatment plan should be developed prior to the procedure, starting with the selection of the target lobe, based on disease distribution and presence or absence of complete interlobar fissures. Based on the information obtained from quantitative CT assessment an individualized approach is recommended when to use the Chartis System and when there is no need for real-time confirmation of collateral ventilation. In this context, a lobe with an adjacent fissure integrity score below $80 \%$ should not be considered eligible for valve treatment, due to a high predictive value of treatment failure based on HRCT alone (48). On the other hand, a patient with a fissure integrity score greater than $95 \%$ next to the target lobe may be offered valve treatment with a high success rate, the Chartis assessment being optional in those cases. If the fissure integrity is between $80 \%$ and $95 \%$, the Chartis System is currently recommended to assess collateral ventilation in real time, as the CT scan does not reliable rule in or rule out patients who may benefit from valve therapy (48).

Both the Chartis procedure and subsequent placement of valves are ideally performed during the same bronchoscopic procedure. The procedure itself can be performed during conscious sedation or general anesthesia (43). Absence of collateral ventilation is confirmed by a gradual decrease and subsequent cessation of expiratory airway flow in addition to a corresponding increase in resistance. Occasionally, testing for collateral ventilation in the target lobe can be inconclusive due to coughing artifacts, mucus plugs, obstruction of the catheter tip by an airway wall, and/ or the "low-flow" or "collapse" phenomenon. The latter is a sudden stop of flow during Chartis assessment, as a consequence of dynamic expiratory airway collapse in emphysema (49). Clinical studies have reported this to occur in about $20 \%$ of patients undergoing Chartis assessment, with a larger proportion in the lower lobes $(27,48)$. Under such circumstances, the ipsilateral lobe may be evaluated as a surrogate for absence of collateral ventilation in the target lobe (43). This is straightforward for the left lower lobe, because there is only one interlobar fissure. For measurements of the right upper or lower lobe, the right middle lobe needs to be plugged temporarily; using a regular balloon catheter or another occluding device, and use the Chartis balloon in either upper or lower lobe. In patients with a collapse phenomenon in either lobe, however, decisions should be based on fissure analysis. In a study by Gesierich et al. (49), most of the collapse phenomenon patients with complete fissures that received EBV responded well to treatment, but the entire collapse phenomenon patients with fissure defects treated were nonresponders.

With respect to the actual treatment, both valve types, the EBV and the IBV, can be implanted by a delivery catheter through the working channel of a $2.8-\mathrm{mm}$ or greater bronchoscope. Different sizes of the valves are available to ensure lobar occlusion despite variable airway anatomy leading to the target lobe (Figure 1). Furthermore, there have been advancements in catheter technology to facilitate valve placement in difficult to treat segments.

\section{Follow-up care}

Post-valve placement, a chest X-ray should be performed within 4 and 24 hours after the procedure. Cough suppression can be helpful for patient comfort in the first few days. Some authors furthermore recommend bed rest for at least 48 hours to reduce the risk of pneumothorax as mentioned earlier (41). Significant volume reduction or atelectasis of the treated lobe may be observed within the first few days, although in some patients it may take up to several weeks (43). Prior to discharge the patient should be informed about the potential risks associated with valve treatment. In most cases, the complications can be managed by the patient's primary care pulmonologist, but coordinated and collaborative communication with the treating center is recommended. A follow-up schedule at 1, 3, 6 months and yearly after the procedure has been recommended to maintain surveillance of patient outcomes and possible complications (43).

If there is no evidence of volume reduction on X-ray and/or improvements in lung function (lung volumes) within the first three months, a low dose CT scan should be performed to ensure correct valve positioning and lobar occlusion. A repeat bronchoscopy may be necessary to replace valves that do not provide sufficient segmental or subsegmental occlusion. Also, patients with a persistent cough, hemoptysis, and/or pneumonia in the target lobe may undergo bronchoscopy to check for valve migration, granulation tissue, or secretion obstruction, potentially prompting (temporary) valve removal.

\section{Discussion points}

Beyond the scientific data and clinical recommendations presented above there are a number of aspects related to 
valve therapy that deserve further attention, some of which are outlined below.

\section{Do improvements in lung function and exercise capacity translate into changes in physical activity after valve treatment?}

Hartman et al. (50) thus studied physical activity, measured by a triaxial accelerometer, in 43 patients with severe COPD at baseline and 6-month follow-up after EBV treatment and compared with standard medical care in a RCT. After 6 months, the valve treatment group had significantly improved step counts and locomotion time per day compared to controls. In the absence of long term survival data from RCTs, yet, this piece of scientific work becomes particularly important to patients, physicians, and health-care payers alike, as measurements of physical activity are considered the strongest independent predictors of COPD related morbidity and mortality.

\section{Is there a long-term survival benefit associated with valve therapy?}

To date survival data for valve treated patients is rather scarce. Two published studies, both with rather small sample sizes, reported on functional outcomes and survival rates beyond 12 months. Venuta et al. (51) followed 33 patients with severe emphysema with a median postinterventional follow-up of 32 months. Patients with complete interlobar fissures (as a surrogate for successful valve therapy and functional benefits) treated with valves demonstrated a statistically significant survival benefit compared with patients with incomplete interlobar fissures. Hopkinson et al. (52) similarly reported on 19 patients treated with valves, of which five patients developed atelectasis after the procedure and none of them died during a follow-up of around 7 years, as opposed to a $57 \%$ mortality rate in those without radiological signs of atelectasis. These findings have been recently confirmed in another retrospective study of 449 patients who underwent valve treatment at an experienced institution in Germany (53). Patients were followed for a mean time of 37 months, of which about $30 \%$ developed complete lobar atelectasis. Patients with atelectasis in that study had a statistically significant survival benefit compared to patients without atelectasis (5-year survival rate $63 \%$ vs. $44 \%$ ). Thus, on the basis of nonrandomized case series there appears to be a survival benefit for those patients with evidence of "significant" volume reduction after valve therapy, albeit the previously used MCID of $350 \mathrm{~mL}$ TLVR has been questioned recently. Using an anchor-based method on data from more than 300 patients with severe emphysema from two valve treatment trials, Wellling and co-workers (54) identified a threshold of at $560-\mathrm{mL}$ or greater TLVR to be required for a clinically meaningful benefit. Alternatively, a post-hoc analysis of the VENT study observed a relationship between the proportional magnitude of TLVR and clinical benefits (55). Patients with $50 \%$ or greater TLVR in that report had statistically significant improvements in the BODE index, another strong predictor of long-term COPD mortality. The latter observation is consistent with results from recent trials $(35,56)$. In summary, given the aforementioned survival benefits in patients with atelectasis, it appears reasonable to aim for a larger magnitude of volume reduction, in order to "deflate" the treated lung as much as possible and thereby achieving the neuromechanical and ventilatory benefits associated with the procedure.

\section{How will valve therapy perform in a real-life environment?}

Transferring treatment success rates of a novel technology from clinical trials into real-life is frequently challenging for various reasons. The LIVE study aimed to provide such an information using collected data from more than 50 different treatment centers, that participated in a prospective registry in Germany (57). Six-month follow-up data were reported for 321 treated patients with a mean predicted $\mathrm{FEV}_{1}$ of $31 \%$ and $\mathrm{RV}$ of $252 \%$ at baseline, respectively. The $\mathrm{FEV}_{1}$ increased by $100 \mathrm{~mL}$ and $\mathrm{RV}$ decreased by $420 \mathrm{~mL}$ after treatment, thus providing efficacy results comparable to those observed in RCTs. Equally important, the number of serious adverse event (SAEs) in that report demonstrated an acceptable safety pattern with pneumothorax rates and COPD exacerbation frequency observed within the range of previous clinical trials. It can therefore be assumed that valve therapy can be successfully performed in clinical routine at dedicated treatment centers.

\section{Is valve therapy cost-effective?}

As health-care payers are increasingly more restrictive, any intervention in COPD needs to demonstrate healtheconomic benefits. There is only limited data available for valve therapy. Pietzsch and co-workers (58) attempted to provide cost-effectiveness data for EBV therapy compared 
to medical management, using Germany as a reference country and calculations based on the high-responder subgroup analysis from the VENT study. Therapy related and clinical event costs were weighed against qualityadjusted life years (QALYs) gained and direct medial costs of the disease. Higher upfront costs for the EBV cohort related to procedural and postprocedural costs were in part compensated by savings in the subsequent years that resulted from lower annual treatment costs compared with a control cohort. The projected EBV-associated 5-year gain of 0.24 QALYs was reportedly higher than the QALY gains estimated in analyses of pharmaceutical interventions, but lower than the gains projected for continuous oxygen therapy and lung transplantation. Obviously more studies are needed to convince health-care budget representatives unanimously to reimburse valve treatment for patients with emphysema.

\section{Conclusion and final remarks}

Based on the above-mentioned data, valve therapy has now been included in the most recent Global Strategy document for the management of COPD and should therefore be considered as a therapeutic option in selected patients with symptomatic emphysema (59). Nevertheless, a number of questions have not been addressed in this review, predominantly due to the lack of scientific data. As such, there is no published data from RCTs comparing the two different types of valves (EBV vs. IBV) on the market, or valves compared with some of the other endoscopic interventions, such as coils, steam, or sealant. Similarly, it remains unknown whether valve treatment in selected patients may outperform LVRS in terms of safety, efficacy, and/or patient preference. There also has been a debate as to the need for pre- and/or post-interventional pulmonary rehabilitation within the clinical community, as few countries have the respective resources and infrastructure to offer both. While it may be hypothesized that rehabilitation prior to the procedure may potentially reduce the periprocedural risk profile and promote postprocedural recovery, postprocedural rehab may be more efficient, due to improvements in work of breathing as a consequence of lung deflation. Finally, future studies may also provide additional information on potential extrapulmonary outcomes of valve therapy, such as effects on comorbidities (metabolic disorders, osteoporosis, reflux disease), depression and anxiety, and/or sleep physiology and sleep quality.

\section{Acknowledgements}

None.

\section{Footnote}

Conflicts of Interest: The author declares having received speaker and/or consultancy fees from Pulmonx and Olympus/Spiration in the past 5 years.

\section{References}

1. Sciurba FC, Rogers RM, Keenan RJ, et al. Improvement in pulmonary function and elastic recoil after lungreduction surgery for diffuse emphysema. $\mathrm{N}$ Engl J Med 1996;334:1095-9.

2. Fishman A, Martinez F, Naunheim K, et al. National Emphysema Treatment Trial Research Group: A randomized trial comparing lung-volume-reduction surgery with medical therapy for severe emphysema. $\mathrm{N}$ Engl J Med 2003;348:2059-73.

3. Naunheim KS, Wood DE, Krasna MJ, et al. Predictors of operative mortality and cardiopulmonary morbidity in the National Emphysema Treatment Trial Research Group: Predictors of operative mortality and cardiopulmonary morbidity in the National Emphysema Treatment Trial. J Thorac Cardiovasc Surg 2006;131:43-53.

4. Valipour A, Burghuber OC. An update on the efficacy of endobronchial valve therapy in the management of hyperinflation in patients with chronic obstructive pulmonary disease. Ther Adv Respir Dis 2015;9:294-301.

5. Zoumot Z, LoMauro A, Aliverti A, et al. Lung Volume Reduction in Emphysema Improves Chest Wall Asynchrony. Chest 2015;148:185-95.

6. Hopkinson NS, Toma TP, Hansell DM, et al. Effect of bronchoscopic lung volume reduction on dynamic hyperinflation and exercise in emphysema. Am J Respir Crit Care Med 2005;171:453-60.

7. Coxson HO, Nasute Fauerbach PV, Storness-Bliss C, et al. Computed tomography assessment of lung volume changes after bronchial valve treatment. Eur Respir J 2008;32:1443-50.

8. Pizarro C, Ahmadzadehfar H, Essler M, et al. Effect of endobronchial valve therapy on pulmonary perfusion and ventilation distribution. PLoS One 2015;10:e0118976.

9. Eberhardt R, Gerovasili V, Kontogianni K, et al. Endoscopic lung volume reduction with endobronchial valves in patients with severe emphysema and established 
pulmonary hypertension. Respiration 2015;89:41-8.

10. Jörgensen K, Houltz E, Westfelt U, et al. Effects of lung volume reduction surgery on left ventricular diastolic filling and dimensions in patients with severe emphysema. Chest 2003;124:1863-70.

11. Pizarro C, Schueler R, Hammerstingl C, et al. Impact of endoscopic lung volume reduction on right ventricular myocardial function. PLoS One 2015;10:e0121377.

12. Tschernko EM, Wisser W, Wanke T, et al. Changes in ventilatory mechanics and diaphragmatic function after lung volume reduction surgery in patients with COPD. Thorax 1997:52:545-50.

13. Kubo K, Koizumi T, Fujimoto K, et al. Effects of lung volume reduction surgery on exercise pulmonary hemodynamics in severe emphysema. Chest 1998;114:1575-82.

14. Sciurba FC, Ernst A, Herth FJ, et al. A randomized study of endobronchial valves for advanced emphysema. N Engl J Med 2010;363:1233-44.

15. Gompelmann D, Eberhardt R, Herth FJ. Collateral ventilation. Respiration. 2013;85:515-20.

16. Koster TD, Slebos DJ. The fissure: interlobar collateral ventilation and implications for endoscopic therapy in emphysema. Int J Chron Obstruct Pulmon Dis 2016;11:765-73.

17. Herth FJ, Noppen M, Valipour A, et al. Efficacy predictors of lung volume reduction with Zephyr valves in a European cohort. Eur Respir J 2012;39:1334-42.

18. Eberhardt R, Herth FJ, Radhakrishnan S, et al. Comparing clinical outcomes in upper versus lower lobe endobronchial valve treatment in severe emphysema. Respiration 2015;90:314-20.

19. Ninane V, Geltner C, Bezzi M, et al. Multicentre European study for the treatment of advanced emphysema with bronchial valves. Eur Respir J 2012;39:1319-25.

20. Sterman DH, Mehta AC, Wood DE, et al. A multicenter pilot study of a bronchial valve for the treatment of severe emphysema. Respiration 2010;79:222-33.

21. Eberhardt R, Gompelmann D, Schuhmann M, et al. Complete unilateral vs partial bilateral endoscopic lung volume reduction in patients with bilateral lung emphysema. Chest 2012;142:900-8.

22. Koenigkam-Santos M, Puderbach M, Gompelmann D, et al. Incomplete fissures in severe emphysematous patients evaluated with MDCT: incidence and interobserver agreement among radiologists and pneumologists. Eur J Radiol 2012;81:4161-6.

23. Koenigkam-Santos M, de Paula WD, Owsijewitsch M, et al. Incomplete pulmonary fissures evaluated by volumetric thin-section CT: semi-quantitative evaluation for small fissure gaps identification, description of prevalence and severity of fissural defects. Eur J Radiol 2013;82:2365-70.

24. van Rikxoort EM, Goldin JG, Galperin-Aizenberg M, et al. A method for the automatic quantification of the completeness of pulmonary fissures: evaluation in a database of subjects with severe emphysema. Eur Radiol 2012;22:302-9.

25. Wielpütz MO, Bardarova D, Weinheimer O, et al. Variation of densitometry on computed tomography in COPD--influence of different software tools. PLoS One 2014;9:e112898.

26. Aljuri N, Freitag L. Validation and pilot clinical study of a new bronchoscopic method to measure collateral ventilation before endobronchial lung volume reduction. J Appl Physiol (1985) 2009;106:774-83.

27. Herzog D, Thomsen C, Poellinger A, et al. Outcomes of Endobronchial Valve Treatment Based on the Precise Criteria of an Endobronchial Catheter for Detection of Collateral Ventilation under Spontaneous Breathing. Respiration 2016;91:69-78.

28. Herth FJ, Eberhardt R, Gompelmann D, et al. Radiological and clinical outcomes of using Chartis to plan endobronchial valve treatment. Eur Respir J 2013;41:302-8.

29. Reymond E, Jankowski A, Pison C, et al. Prediction of lobar collateral ventilation in 25 patients with severe emphysema by fissure analysis with CT. AJR Am J Roentgenol 2013;201:W571-5.

30. Gompelmann D, Eberhardt R, Slebos DJ, et al. Diagnostic performance comparison of the Chartis System and highresolution computerized tomography fissure analysis for planning endoscopic lung volume reduction. Respirology 2014;19:524-30.

31. Davey C, Zoumot Z, Jordan S, et al. Bronchoscopic lung volume reduction with endobronchial valves for patients with heterogeneous emphysema and intact interlobar fissures (the BeLieVeR-HIFi study): a randomised controlled trial. Lancet 2015;386:1066-73.

32. Zoumot Z, Davey C, Jordan S, et al. Endobronchial valves for patients with heterogeneous emphysema and without interlobar collateral ventilation: open label treatment following the BeLieVeR-HIFi study. Thorax 2017;72:277-9.

33. Klooster K, ten Hacken NH, Hartman JE, et al. Endobronchial valves for emphysema without interlobar collateral ventilation. N Engl J Med 2015;373:2325-35.

34. Klooster K, Hartman JE, Ten Hacken NH, et al. One-Year Follow-Up after Endobronchial Valve Treatment in Patients 
with Emphysema without Collateral Ventilation Treated in the STELVIO Trial. Respiration 2017;93:112-21.

35. Kemp SV, Slebos DJ, Kirk A, et al. A Multicenter Randomized Controlled Trial of Zephyr Endobronchial Valve Treatment in Heterogeneous Emphysema (TRANSFORM). Am J Respir Crit Care Med 2017;196:1535-43.

36. Criner GJ, Sue R, Wright S, et al. A Multicenter RCT of Zephyr® Endobronchial Valve Treatment in Heterogeneous Emphysema (LIBERATE). Am J Respir Crit Care Med 2018. [Epub ahead of print].

37. Valipour A, Slebos DJ, Herth F, et al. Endobronchial valve therapy in patients with homogeneous emphysema: results from the IMPACT study. Am J Respir Crit Care Med 2016;194:1073-82.

38. Wang G, Li S, Wang C, et al. The REACH study, a randomized controlled trial assessing the safety and effectiveness of the Spiration Valve System endobronchial therapy for severe emphysema: 12 month follow-up results. Eur Respir J 2017;50:OA1465.

39. Valipour A, Slebos DJ, de Oliveira HG, et al. Expert statement: pneumothorax associated with endoscopic valve therapy for emphysema - potential mechanisms, treatment algorithm, and case examples. Respiration 2014;87:513-21.

40. van Geffen WH, Klooster K, Hartman JE, et al. Pleural Adhesion Assessment as a predictor for pneumothorax after Endobronchial Valve Treatment. Respiration 2017;94:224-31.

41. Herzog D, Poellinger A, Doellinger F, et al. Modifying post-operative medical care after EBV implant may reduce pneumothorax incidence. PLoS One 2015;10:e0128097.

42. Tuleta I, Pizarro C, Molitor E, et al. Recurrent Chronic Obstructive Pulmonary Disease Exacerbations after Endobronchial Valve Implantation are associated with the presence of pseudomonas aeruginosa. Respiration 2016;91:510-6.

43. Slebos DJ, Shah PL, Herth FJ, et al. Endobronchial Valves for Endoscopic Lung Volume Reduction: Best Practice Recommendations from Expert Panel on Endoscopic Lung Volume Reduction. Respiration 2017;93:138-50.

44. Darwiche K, Karpf-Wissel R, Eisenmann S, et al. Bronchoscopic Lung Volume Reduction with Endobronchial Valves in Low-FEV1 Patients. Respiration 2016;92:414-9.

45. Valipour A, Shah PL, Gesierich W, et al. Patterns of Emphysema Heterogeneity. Respiration 2015;90:402-11.

46. Lim HJ, Weinheimer O, Wielpütz MO, et al. Fully
Automated Pulmonary Lobar Segmentation: Influence of Different Prototype Software Programs onto Quantitative Evaluation of Chronic Obstructive Lung Disease. PLoS One 2016;11:e0151498.

47. Thomsen C, Theilig D, Herzog D, et al. Lung perfusion and emphysema distribution affect the outcome of endobronchial valve therapy. Int J Chron Obstruct Pulmon Dis 2016;11:1245-59.

48. Koster TD, van Rikxoort EM, Huebner RH, et al. Predicting Lung Volume Reduction after Endobronchial Valve Therapy Is Maximized Using a Combination of Diagnostic Tools. Respiration 2016;92:150-7.

49. Gesierich W, Samitas K, Reichenberger F, et al. Collapse phenomenon during Chartis collateral ventilation assessment. Eur Respir J 2016;47:1657-67.

50. Hartman JE, Klooster K, Slebos DJ, et al. Improvement of physical activity after endobronchial valve treatment in emphysema patients. Respir Med 2016;117:116-21.

51. Venuta F, Anile M, Diso D, et al. Long-term follow-up after bronchoscopic lung volume reduction in patients with emphysema. Eur Respir J 2012;39:1084-9.

52. Hopkinson NS, Kemp SV, Toma TP, et al. Atelectasis and survival after bronchoscopic lung volume reduction for COPD. Eur Respir J 2011;37:1346-51.

53. Gompelmann D, Benjamin N, Bischoff E, et al. Survival after endoscopic valve therapy in patients with severe emphysema. Eur Respir J 2016;48:OA472.

54. Welling JBA, Hartman JE, van Rikxoort EM, et al. Minimal important difference of target lobar volume reduction after endobronchial valve treatment for emphysema. Respirology 2018;23:306-10.

55. Valipour A, Herth FJ, Burghuber OC, et al. Target lobe volume reduction and COPD outcome measures after endobronchial valve therapy. Eur Respir J 2014;43:387-96.

56. Klooster K, Hartman JE, Ten Hacken NHT, et al. Improved Predictors of Survival after Endobronchial Valve Treatment in Patients with Severe Emphysema. Am J Respir Crit Care Med 2017;195:1272-4.

57. Skowasch D, Fertl A, Schwick B, et al. A long-term followup investigation of endobronchial valves in emphysema (the LIVE Study): study protocol and six-month interim analysis results of a prospective five-year observational study. Respiration 2016;92:118-26.

58. Pietzsch JB, Garner A, Herth FJ. Cost-effectiveness of endobronchial valve therapy for severe emphysema: a model-based projection based on the VENT study. 
Respiration 2014;88:389-98.

59. Vogelmeier CF, Criner GJ, Martinez FJ, et al. Global Strategy for the Diagnosis, Management, and Prevention of Chronic Obstructive Lung Disease 2017 Report:

Cite this article as: Valipour A. Valve therapy in patients with emphysematous type of chronic obstructive pulmonary disease (COPD): from randomized trials to patient selection in clinical practice. J Thorac Dis 2018;10(Suppl 23):S2780-S2796. doi: 10.21037/jtd.2018.08.86
GOLD Executive Summary. Eur Respir J 2017;49. doi: 10.1183/13993003.00214-2017. Erratum in: Eur Respir J $2017 ; 49$. 